\title{
Overexpression of septin-7 inhibits melatonin-induced cell apoptosis in human fetal osteoblastic cells via suppression of endoplasmic reticulum stress
}

\author{
XIAOTONG MENG, YUE ZHU, LIN TAO, SICHAO ZHAO and SHUI QIU \\ Department of Orthopaedics, First Affiliated Hospital of China Medical University, Shenyang, Liaoning 110001, P.R. China
}

Received August 25, 2017; Accepted December 12, 2017

DOI: $10.3892 / \mathrm{mmr} .2018 .8449$

\begin{abstract}
Our previous study demonstrated that melatonin could induce apoptosis in the human fetal osteoblastic (hFOB) 1.19 cell line via induction of endoplasmic reticulum stress (ERS), and recent studies have demonstrated that the expression of septin-7 (SEPT7) exhibits a positive correlation with the concentration of melatonin. Western blotting demonstrated the expression level of SEPT7 was significantly upregulated in a dose-dependent manner following treatment with differing concentrations of melatonin compared with the control groups, which did not receive any treatment. The expression of proteins associated with cell apoptosis and endoplasmic reticulum stress (ERS; pro-caspase-3, cleaved caspase-3, C/EBP-homologous protein, $78 \mathrm{kDa}$ glucose-regulated protein and phosphorylated-eukaryotic translation initiation factor $2 \alpha$ ) were decreased following transfection with SEPT7 overexpression plasmid and increased following transfection with SEPT7 small interfering RNA compared with the control groups. The results of the present study suggest that SEPT7 inhibits melatonin-induced cell apoptosis via suppression of ERS.
\end{abstract}

\section{Introduction}

Septins belong to a class of cytoskeletal proteins with GTPase activity, which can form intracellular filamentous scaffolds. Previous studies have demonstrated that septins are involved in numerous biological processes, such as cell mitosis, polarity determination, vesicle trafficking and apoptosis (1-4). In addition, a previous study revealed that septin-7 (SEPT7), a member of septin family, can suppress cell cycle progression in yeast via its highly homologous cDNA sequence to cell division control protein 10 (5). Furthermore, a previous study

Correspondence to: Dr Yue Zhu, Department of Orthopaedics, First Affiliated Hospital of China Medical University, 155 Nan Jing Bei Street, Shenyang, Liaoning 110001, P.R. China

E-mail: zhuyuedr@163.com

Key words: apoptosis, endoplasmic reticulum stress, human osteoblastic cells line hFOB 1.19, melatonin, septin-7 revealed that SEPT7 exerts a suppressive effect on glioma cell proliferation and invasion and induces apoptosis, and it has been suggested that SEPT7 exerted suppressive effects on the growth and invasion of glioma cells, inducing cell apoptosis, which suggested that SEPT7 is a glioma suppressor gene (6). Zhang et al (7) demonstrated that the silencing of SEPT7 inhibits cell proliferation and apoptosis in human breast cancer cells. In addition, it has been demonstrated that miR-127 can inhibit cell proliferation via suppression of SEPT7 expression in hepatocellular carcinoma cells (HCC), which suggests that SEPT7 promotes cell proliferation and inhibits apoptosis (8). Therefore, it may be suggested that in tumor-associated cells, SEPT7 may exhibit an inhibitory and promotive effect on cell apoptosis; thus the exact molecular mechanism of SEPT7 has not yet been determined. Our previous study demonstrated that melatonin can induce cell apoptosis in the human fetal osteoblastic cell line hFOB 1.19 by activating the phosphorylated extracellular signal regulated kinase (p-ERK)-p-eukaryotic translation initiation factor $2 \alpha$ (eIF2 $\alpha$ )-activating transcription factor 2 signal transduction pathway (9), and ERS may mediate this process. In the present study, in vitro experiments were performed to further investigate whether SEPT7 inhibits melatonin-induced apoptosis or whether it acts as a potential target for melatonin in the hFOB 1.19 cell line. In the present study, in vitro experiments were performed following the construction and transfection of a SEPT7 overexpression plasmid into hFOB 1.19 cells, and apoptosis/ERS associated proteins including (p-eIF2 $\alpha$ ), $78 \mathrm{kDa}$ glucose-regulated protein (GRP78), C/EBP-homologous protein (CHOP), procaspase-3 and cleaved caspase-3 were investigated to reveal whether SEPT7 has an inhibitory or stimulative effect in melatonin-induced apoptosis, or whether SPET7 acts as a potential target of melatonin in hFOB 1.19 cells.

\section{Materials and methods}

Cell culture and reagents. The hFOB 1.19 cell line, provided by the Department of Biochemistry and Molecular Biology, the Mayo Clinic (Rochester, MN, USA) (10) was maintained in a 1:1 mixture of Dulbecco's modified Eagle's medium (DMEM) and F12 medium without phenol red (HyClone, Laboratories; GE Healthcare Life Sciences, Logan, UT, USA). Cells were supplemented with $10 \%$ fetal bovine serum (FBS; Clark Bioscience, Richmond, VA, USA) and maintained 
in an atmosphere of $5 \% \mathrm{CO}_{2}$ and at a temperature of $37^{\circ} \mathrm{C}$. Medium was replaced with fresh medium every two days. Cells were utilized in passages 8-11. Melatonin dissolved in $0.2 \%$ dimethyl sulfoxide (DMSO) or vehicle treatment with $0.2 \%$ DMSO (in culture medium, DMEM and F12) was performed $37^{\circ} \mathrm{C}$ with $10 \% \mathrm{FBS}$. Melatonin was obtained from Sigma-Aldrich; Merck KGaA (Darmstadt, Germany). Primary monoclonal antibodies against GRP78 (cat. no. ab21685), caspase-3 (cat. no. ab90437), p-eIF2 $\alpha$ (cat. no. ab4837), SEPT7 (cat. no. ab186021), CHOP (cat. no. 194533) and goat anti-rabbit secondary antibodies (cat. no. ab6721) were all purchased from Abcam (Cambridge, MA, USA).

Overexpression plasmid construction. The SEPT7 fragment was cloned from normal human cDNA from hFOB 1.19 cells into GV230 plasmids (200 ng; Shanghai GeneChem Co., Ltd., Shanghai, China) between XhoI/KpnI sites to overexpress SEPT7 in hFOB 1.19 cells. Full-length SEPT7 gene (4,377 bp; GenBank NM_001011553) was amplified by polymerase chain reaction (PCR). The PCR primers used were as follows: Forward, 5'-CTGCTCACAATAGTTGAT ACCCC-3' and reverse, 5'-TGTTCACTCGTGATTCTG CATT-3'. The PrimeSTAR HS DNA polymerase, was also obtained from Shanghai GeneChem Co., Ltd., and cycled for 30 cycles following initial denaturation $\left(98^{\circ} \mathrm{C}\right.$ for $5 \mathrm{~min}$ ) with the following parameters: $72^{\circ} \mathrm{C}$ for $8 \mathrm{~min}$. Following enzyme digestion (Exnase ${ }^{\mathrm{TM}}$ II; $1 \mu \mathrm{l}$ ) using ClonExpress II One Step Cloning kit (Vazyme Biotech Co., Ltd., Nanjing, China) and sequencing, the PCR product was cloned into the XhoI/KpnI sites of the GV230 expression vector. The recombinant GV230-SEPT7 plasmid was confirmed via endonuclease digestion and DNA sequencing (Shanghai GeneChem Co., Ltd.) prior to transfection into hFOB 1.19 cells using Lipofectamine $2000^{\circledR}$ (Invitrogen; Thermo Fisher Scientific, Inc., Waltham, MA, USA). In addition, the GV230 vector (used as a negative control) was also transfected into cells using Lipofectamine $2000^{\circledR}$.

SEPT7 small interfering (si)RNA transfection. Cells were cultured in DMEM and F12 medium supplemented with $10 \%$ FBS (Clark Bioscience) in a humidified incubator at $37^{\circ} \mathrm{C}$ and $5 \% \mathrm{CO}_{2}$. At 70-80\% confluence, cells were transfected with SEPT7 small interfering (si)RNA (60 nM; sense, 5'-CGA CUACAUUGAUAGUAAAUU-3' and antisense, 5'-UUUACU AUCAAUGUAGUCGAU-3'; (Shanghai Genechem Co., Ltd.) using Lipofectamine ${ }^{\circledR} 2000$ according to the manufacturer's protocol (Invitrogen; Thermo Fisher Scientific, Inc.). Overall, there were three control groups: Blank control (medium only), transfection reagent control (to eliminate the toxic influence of Lipofectamine 2000, and the influence of Lipofectamine 2000 on the expression of the target gene) and scrambled siRNA control (5'-GAAATTTATAACGATCAGTCT-3').

Western blotting. Following treatment, proteins were extracted from hFOB 1.19 cells via incubation with radioimmunoprecipitation assay lysis buffer (Beyotime Institute of Biotechnology, Shanghai, China) for $30 \mathrm{~min}$ at $4^{\circ} \mathrm{C}$. The supernatant containing total protein was harvested and proteins were quantified using the bicinchoninic acid method. Aliquots containing $50 \mu \mathrm{g}$ protein per lane were separated on a $12 \%$ gel using SDS-PAGE and then transferred to polyvinylidene fluoride membranes at $60 \mathrm{~V}$ for $2 \mathrm{~h}$ at $4^{\circ} \mathrm{C}$. Subsequently, membranes were soaked in $5 \%$ blocking buffer, containing $25 \mathrm{mg}$ bovine serum albumin (Beyotime Institute of Biotechnology) in Tris-buffered saline (TBS) buffer to final volume of 0.5 liters, at $4^{\circ} \mathrm{C}$ for $2 \mathrm{~h}$. Proteins were then incubated with primary antibodies against to GRP78 (cat. no. ab21685), caspase-3 (cat. no. ab90437), p-eIF2 $\alpha$ (cat. no. ab4837), SEPT7 (cat. no. ab186021), CHOP (cat. no. 194533) and $\beta$-actin (cat. no. ab8226) were purchased from Abcam (Cambridge, MA, USA), diluted at 1: 5,000 and incubated overnight at $4^{\circ} \mathrm{C}$, followed by incubation with goat anti-rabbit horseradish peroxidase-conjugated secondary antibodies (1:10,000; cat. no. ab6721) for $2 \mathrm{~h}$ at room temperature. The DNR imaging system (DNR Bio-Imaging Systems, Ltd., Jerusalem, Israel) was used to visualize specific bands using the BeyoECL Plus (Beyotime Institute of Biotechnology) protocol, and the optical density of each band was determined using ImageJ software (version 1.51; National Institute of Health, Bethesda, MD, USA). The ratio between target proteins and $\beta$-actin was determined and graphically presented.

Reverse transcription-quantitative polymerase chain reaction (RT-qPCR) assay. Total RNA was extracted from hFOB 1.19 cells using E.Z.N.A. ${ }^{\circledR}$ Total RNA Midi kit (Omega Bio-Tek, Inc., Norcross, GA, USA) according to the manufacturer's protocol. Samples were quantified spectrophotometrically at $260 \mathrm{~nm}$, with 260/280 $\mathrm{nm}$ ratios of 1.8-2.0 considered to be acceptable. RNA quality was confirmed using $1 \%$ agarose gel electrophoresis stained with $1 \mu \mathrm{g} / \mathrm{ml}$ ethidium bromide. qPCR was performed using a LightCycler ${ }^{\circledR} 480$ High-Resolution Melting Master (Roche Diagnostics, Basel, Switzerland) using SYBR Premix Ex Taq ${ }^{\mathrm{TM}}$ II (Takara Biotechnology Co., Ltd., Dalian, China). Specific primers for SEPT7 (forward, 5'-ACGGGTTAGGCTCTTGG-3' and reverse primer, 5'-CAG TGCGTGTCGTGGAGT-3') were obtained from Shanghai GeneChem Co., Ltd. Amplifications were performed in a total volume of $20 \mu \mathrm{l}$ and cycled 40 times following initial denaturation $\left(95^{\circ} \mathrm{C}\right.$ for $\left.30 \mathrm{sec}\right)$ with the following parameters: $95^{\circ} \mathrm{C}$ for $5 \mathrm{sec}$ and $60^{\circ} \mathrm{C}$ for $30 \mathrm{sec}$. $\beta$-actin (forward, $5^{\prime}$-TCCTCCCTG GAGAAGAGCTA-3' and reverse, 5'-TCAGGAGGAGCA ATGATCTTG-3') was used as an internal control. Analysis of the melting curve was performed to further verify the results of RT-qPCR. RT-qPCR data were quantified using the $2^{-\Delta \Delta C q}$ method (11).

Statistical analysis. Data are presented as mean \pm standard error of the mean and a minimum of three independent repeats were performed for each experiment. SPSS software (version 20.0; IBM Corp., Armonk, NY, USA) was used to perform data analysis. Independent t-tests or one-way analysis of variance followed by the Student-Newman-Keuls test were performed to investigate differences between multiple groups. $\mathrm{N}$-fold values of $\leq 0.5$ and $>2$ gene expression compared with control genes were considered to be significant. $\mathrm{P}<0.05$ was considered to indicate a statistically significant difference.

\section{Results}

Expression of SEPT7 increased with the increasing concentration of melatonin. First, western blot analysis revealed that 
in hFOB 1.19 cells, the expression level of SEPT7 was significantly upregulated in a dose-dependent manner following treatment with differing concentrations of melatonin compared with the control groups which did not receive any treatments $(\mathrm{P}<0.05)$ (Fig. 1).

The influence of SEPT7 overexpression and inhibition on melatonin-induced apoptosis and ERS in human hFOB 1.19 cells. In addition, to assess whether SEPT7 affects melatonin-induced apoptosis, the SEPT7 overexpression plasmid and SEPT7 siRNA was constructed and transfected into hFOB 1.19 cells. The expression of SEPT7 in the GV230-SEPT7 transfected group demonstrated a marked increase in expression, and the expression level of SEPT7 in the SEPT7 siRNA transfected group exhibited an obvious decrease compared with the control group (the GV230 vector transfected group; Fig. 2).

Following this, cells were treated with $4 \mathrm{mM}$ melatonin and the expression of proteins associated with cell apoptosis and endoplasmic reticulum stress (ERS) were investigated via western blotting. The results of these analyses revealed that the levels of cleaved caspase-3, C/EBP homologous protein (CHOP), $78 \mathrm{kDa}$ glucose-regulated protein (GRP78) and phosphorylated-eukaryotic translation initiation factor $2 \alpha$ (p-eIF $2 \alpha)$ significantly increased and the expression of pro-caspase- 3 decreased following treatment with melatonin compared with the control group which did not receive any treatments, thus suggesting that the rate of cell apoptosis increased. In addition, in the SEPT7 overexpression $+4 \mathrm{mM}$ melatonin group, the levels of cleaved caspase-3, CHOP, GRP78 and p-eIF2 $\alpha$ were significantly increased compared with the control group, and markedly suppressed compared with the $4 \mathrm{mM}$ melatonin group (Fig. 3).

Finally, either SEPT7 siRNA or control siRNA were transfected into osteoblasts using Lipofectamine ${ }^{\circledR}$ 2000 according to the manufacturer's protocol. There were three control groups: Blank control, transfection reagent control and scramble siRNA control. A rescue experiment was also performed via transfection with the SEPT7 overexpression plasmid (Shanghai Genechem Co., Ltd.). Western blot analysis was then performed to assess the protein levels of CHOP, ATF4, p-eIF2 $\alpha$, pro-caspase- 3 and cleaved caspase- 3 . The results demonstrated that the levels of CHOP, ATF4, p-eIF2 $\alpha$ and cleaved caspase- 3 in the group treated with melatonin in combination with SEPT7 siRNA were increased significantly, compared with those in the control groups (Fig. 4). Following transfection with SEPT7 siRNA, the expression level of SEPT7 decreased significantly (Fig. 2). The SEPT7 overexpression plasmid was then transfected into cells in order to perform the rescue experiment. The results demonstrated that following transfection with the SEPT7 overexpression plasmid the expression level of SEPT7 was increased to a level similar to the $4 \mathrm{mM}$ melatonin-treated group, compared with the control group (Fig. 2). Therefore, the results suggest that silencing of SEPT7 may enhance the intensity of melatonin-induced apoptosis (by affecting the level of apoptosis-related proteins) and ERS in human osteoblasts cell line hFOB 1.19. Therefore concluding that the existence of SEPT7 may be a protective factor in melatonin-induced apoptosis in human osteoblasts cell line hFOB 1.19 .
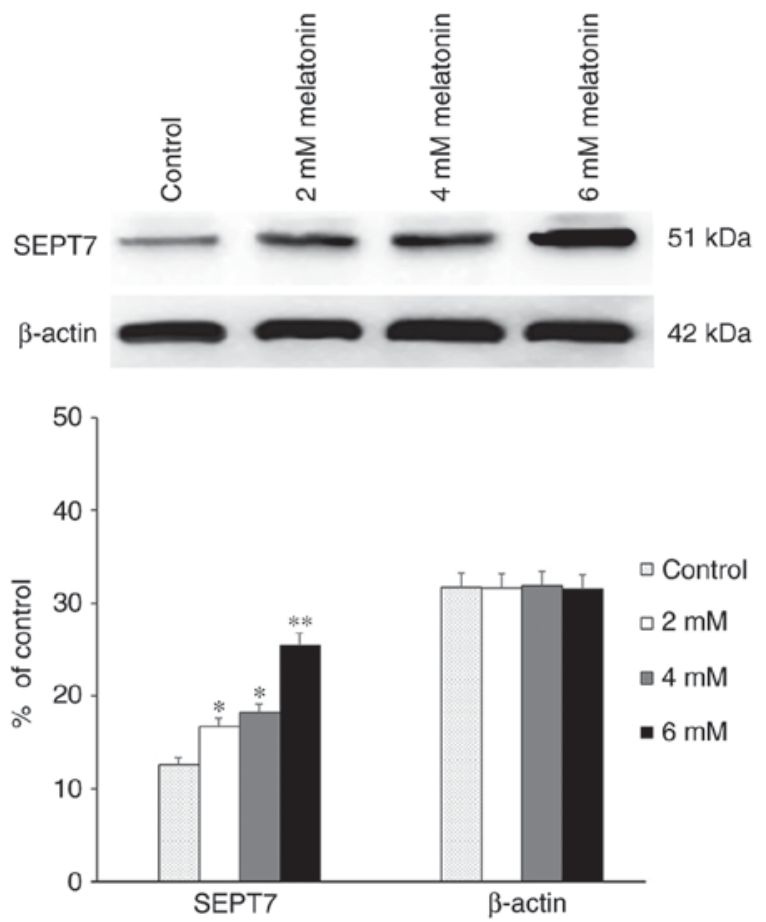

Figure 1. Expression of SEPT7 following treatment with different concentrations of melatonin for $24 \mathrm{~h}$ in human osteoblastic cells line hFOB 1.19. Each bar represents the mean \pm standard error of the mean of three independent experiments. ${ }^{*} \mathrm{P}<0.05$ and ${ }^{* *} \mathrm{P}<0.01$ vs. control cells, which did not receive any treatments. SEPT7, septin-7.

\section{Discussion}

It has previously been demonstrated that melatonin is associated with aging, reproduction, tumor growth and multiple other biological progresses in human cells (12-14). Our previous study revealed that melatonin can regulate cell apoptosis by inducing ERS in hFOB 1.19 cells (9). During melatonin-induced apoptosis, proteins associated with well-established apoptotic pathways, such as CHOP, pro-caspase-3, cleaved caspase-3 and p-eIF $2 \alpha$; were upregulated, and thus may represent indicators for the occurrence and rate of melatonin induced apoptosis.

Septins are a class of super protein families with a molecular weight of 30-65 kDa and have conserved structures. Previous studies have demonstrated that septin proteins are one of the four cytoskeletal components, the other three being tubulin, microfilament and intermediate fibrin $(15,16)$, and have important roles in the transportation of intracellular substances $(17,18)$, the regulation of cell division and the cell cycle $(19,20)$ as well as other physiological processes, such as apoptosis. A previous study demonstrated that under certain conditions, such as in the presence of apoptotic factors, septin 4 (SEPT4) may become detached from the mitochondria and be released into the cytoplasm following binding with an inhibitor of cysteine and aspartic acid proteases, it is then relieved of the inhibitory effect of X-linked inhibitor of apoptosis protein and enhanced the activity of cysteine and aspartic acid proteases afterwards, then finally promotes cell apoptosis (21). However, it has been demonstrated that septin 9 (SEPT9) is overexpressed in numerous types of tumors and also promotes cell proliferation in prostate cancer $(22,23)$. Therefore, the effects of SEPT4 and SEPT9 are opposite and thus the functions of the septin 


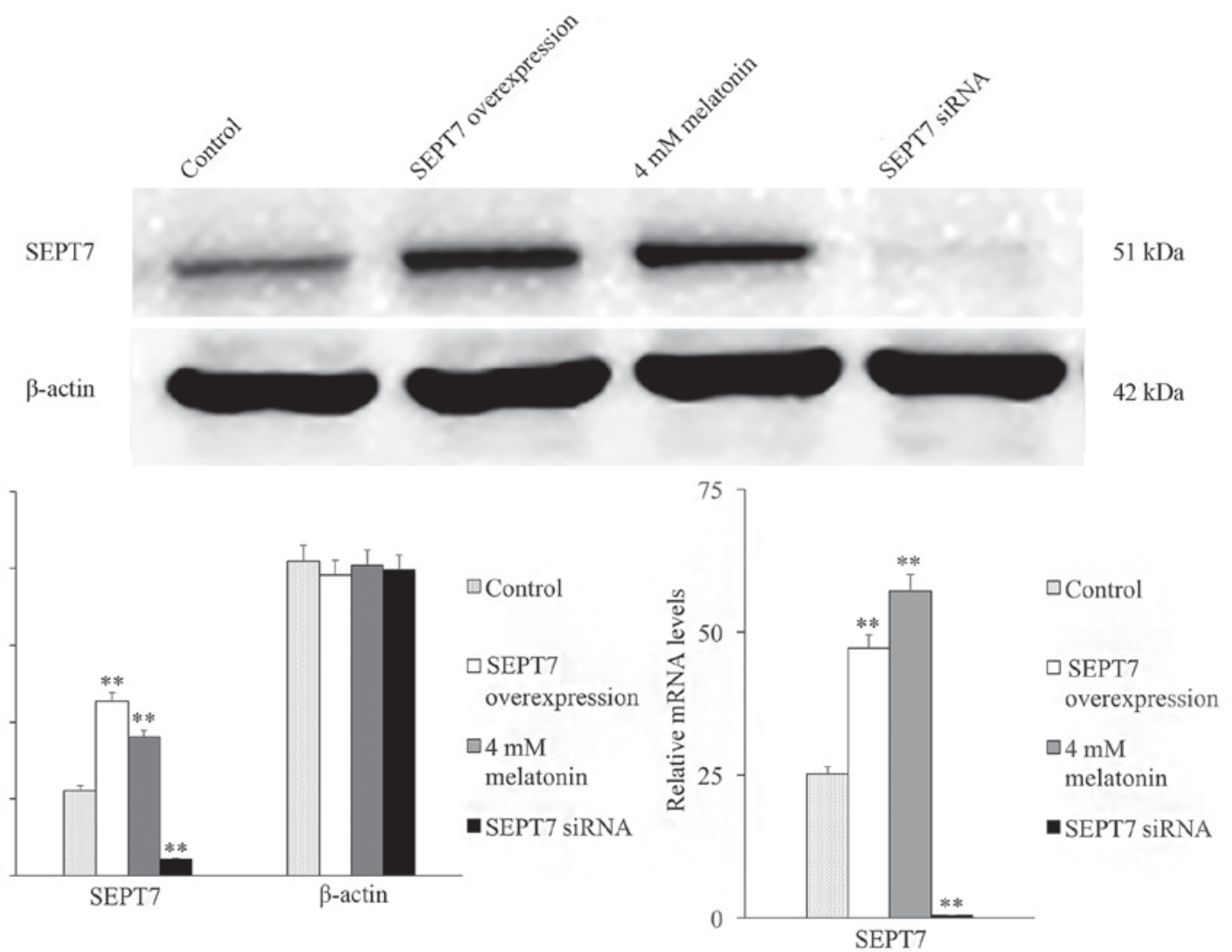

Figure 2. Protein and mRNA expression of SEPT7 following treatment with SEPT7 overexpression plasmid or SEPT7 siRNA in human osteoblastic cells line hFOB 1.19. Each bar represents the mean \pm standard error of the mean of three independent experiments. ${ }^{*} \mathrm{P}<0.05$ and ${ }^{* *} \mathrm{P}<0.01 \mathrm{vs}$. control cells. SEPT7, septin-7; si, small interfering.
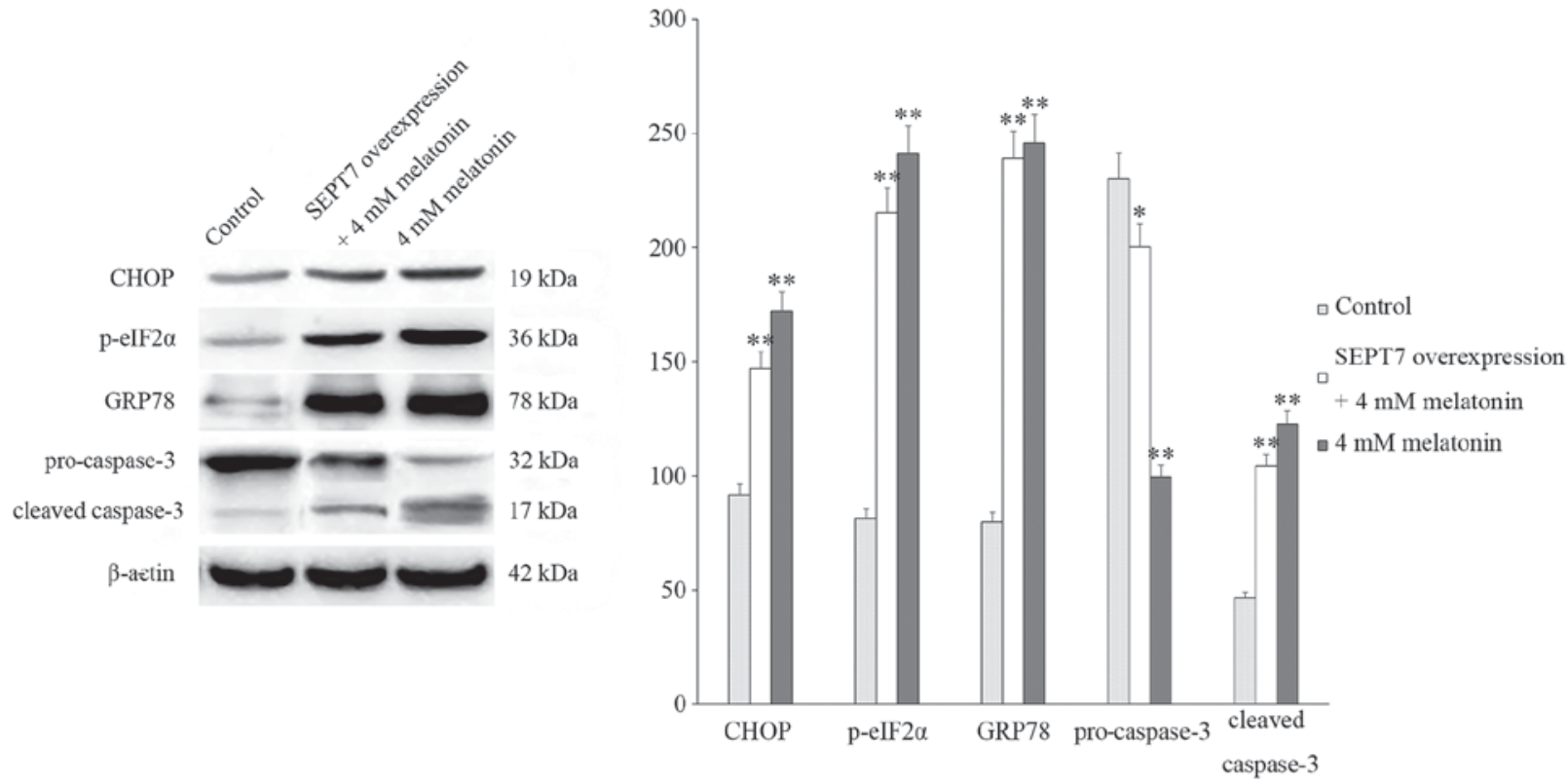

Figure 3. Expression of CHOP, p-eIF2 $\alpha$, GRP78, pro-caspase-3 and cleaved caspase-3 following treatment with $4 \mathrm{mM}$ melatonin alone for $24 \mathrm{~h}$, or after the transfection with the SEPT7 overexpressing plasmid in human osteoblastic cells line hFOB 1.19. Each bar represents the mean \pm standard error of the mean of three independent experiments. ${ }^{*} \mathrm{P}<0.05$ and ${ }^{* *} \mathrm{P}<0.01$ vs. control cells. SEPT7, septin-7; CHOP, C/EBP-homologous protein; p-eIF $2 \alpha$, phosphorylated eukaryotic translation initiation factor $2 \alpha$; GRP78, $78 \mathrm{kDa}$ glucose-regulated protein; si, small interfering.

superfamily associated with cell proliferation and apoptosis are unclear. Considering that septin proteins have been identified in all eukaryotes (15) and that the association between SEPT7 and hFOB 1.19 cells has not been extensively investigated, the present study aimed to investigate whether SEPT7 has a role in melatonin-induced cell apoptosis in hFOB 1.19 cells. The 

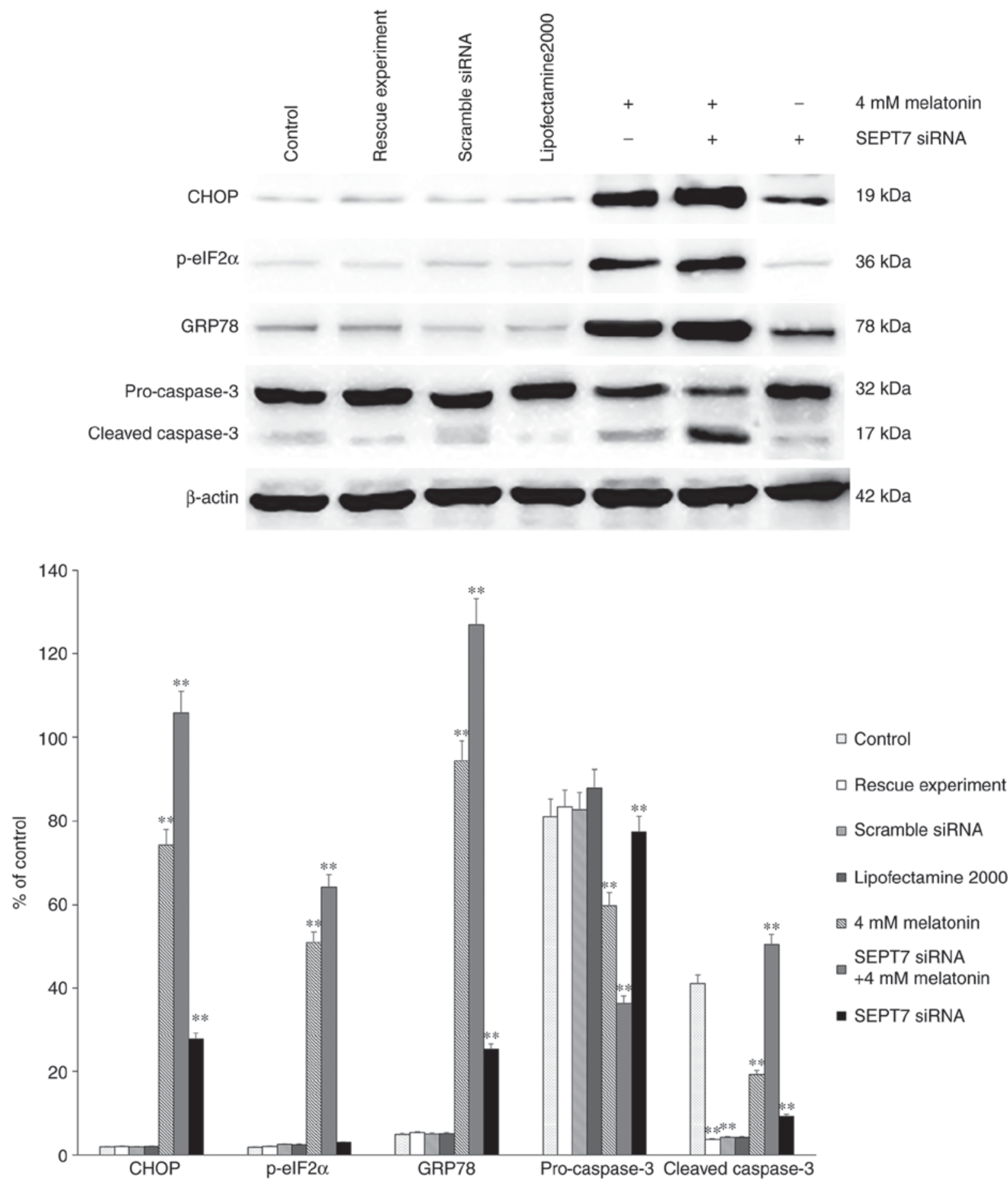

Figure 4. Expression of CHOP, p-eIF2 $\alpha$, GRP78, pro-caspase-3 and cleaved caspase- 3 after treatment with $4 \mathrm{mM}$ melatonin alone for $24 \mathrm{~h}$, or after the transfection of SEPT7 siRNA in human osteoblastic cells line hFOB 1.19. Each bar represents the mean \pm standard error of the mean of three independent experiments. " $\mathrm{P}<0.05$ and ${ }^{* *} \mathrm{P}<0.01$ vs. control cells. SEPT7, septin-7; CHOP, C/EBP-homologous protein; p-eIF2 $\alpha$, phosphorylated eukaryotic translation initiation factor $2 \alpha$; GRP78, $78 \mathrm{kDa}$ glucose-regulated protein; si, small interfering.

results of western blot analysis revealed that the expression of SEPT7 was upregulated in melatonin-treated groups in a dose-dependent manner, which suggests that SEPT7 may be a protective factor in melatonin-induced apoptosis in hFOB 1.19 cells. To further investigate this, a SEPT7 overexpression plasmid was constructed and transfected into hFOB 1.19 cells, and apoptosis-associated proteins CHOP and caspase-3 were investigated via western blot analysis. Results demonstrated the above-mentioned proteins decreased in the SEPT7 overexpression group compared with the $4 \mathrm{mM}$ melatonin treatment group, which can demonstrate that the existence of SEPT7 served a protective role in melatonin-induced apoptosis in human osteoblasts cell line hFOB 1.19. The results therefore suggested that SEPT7 attenuates apoptosis in hFOB 1.19 cells. Transfection with SEPT7-siRNA also demonstrated that SEPT7 has a protective, not a stimulative, role in melatonin-induced cell apoptosis in hFOB 1.19 cells. Previous studies have revealed that SEPT7 inhibits apoptosis via a caspase-independent pathway (24), which is consistent with our conclusions. However overexpression of SEPT7 inhibits cell 
proliferation and induces $\mathrm{G}_{0} / \mathrm{G}_{1}$ phase arrest in human glioma cells, both in vitro and in vivo (25) which is in contrast with our conclusions, the anti-apoptotic effect in normal cells indicates that SEPT7 may serve a protective role in all kinds of cells.

Our previous study (9) have demonstrated that melatonin-induced cell apoptosis in hFOB 1.19 cells is associated with the protein kinase RNA-like endoplasmic reticulum kinase pathway, and the occurrence of ERS was demonstrated via investigation into the expression levels of GRP78 and GRP94. Previous study has suggested that the expression levels of GRP78 and GRP94 are significantly enhanced when ERS occurs (26). Therefore, the present study investigated ERS-associated proteins GRP78 and p-eIF2 $\alpha$; the results revealed that following transfection of the melatonin-treated cells with the SEPT7 siRNA, the expression levels of GRP78 were significantly increased compared with those in SEPT7 siRNA non-transfected melatonin-treated cells. Furthermore, the levels of GRP78 and p-eIF2 $\alpha$ were significantly decreased in the SEPT7 siRNA transfected group compared with the SEPT7 siRNA non-transfected and melatonin-treated cells, which suggests that the intensity of the ERS was decreased compared with the $4 \mathrm{mM}$ melatonin group (Fig. 4). Taken together, it can be suggested that SEPT7 inhibits melatonin-induced cell apoptosis via suppression of ERS.

However, the underlying mechanism associated with the effects of SEPT7 remains unclear. An ongoing study by the authors suggests that this mechanism may be associated with certain miRNAs (MiR-590-3p; Meng et al, unpublished data), and we will investigate this further in later studies.

\section{Acknowledgements}

We would like to thank Dr Subramaniam M (Department of Biochemistry and Molecular Biology, Mayo Clinic) for providing the human fetal osteoblastic cell line hFOB 1.19. In addition, we would like to thank the National Natural Science Foundation of China (grant. no. 81472044) for their financial support and the Shenyang Science and Technology Program-Population and Health Special (17-230-9-04) of Shenyang supported by the Science and Technology Bureau of Shenyang City, Liaoning Province.

\section{References}

1. Beites CL, Xie H, Bowser R and Trimble WS: The septin CDCrel-1 binds syntaxin and inhibits exocytosis. Nat Neurosci 2 434-439, 1999

2. Field CM and Kellogg D: Septins: Cytoskeletal polymers or signalling GTPases? Trends Cell Biol 9: 387-394, 1999.

3. Larisch S, Yi Y, Lotan R, Kerner H, Eimerl S, Tony Parks W, Gottfried Y, Birkey Reffey S, de Caestecker MP, Danielpour D, et al: A novel mitochondrial septin-like protein, ARTS, mediates apoptosis dependent on its P-loop motif. Nat Cell Biol 2: 915-921, 2000.

4. Kartmann B and Roth D: Novel roles for mammalian septins: From vesicle trafficking to oncogenesis. J Cell Sci 114: 839-844, 2001.

5. Hou MS, Liu XB, Cao J and Chen B: SEPT7 overexpression inhibits glioma cell migration by targeting the actin cytoskeleton pathway. Oncol Rep 35: 2003-2010, 2016.

6. Jia ZF, Pu PY, Kang CS, Wang GX, Zhang ZY, Qiu MZ and Huang Q: Influence of SEPT7 on biological characters of glioma cell line TJ905. Zhonghua Wai Ke Za Zhi 45: 1420-1423, 2007 (In Chinese).
7. Zhang NZ, Liu L, Fan N, Zhang Q, Wang W, Zheng M, Ma L, $\mathrm{Li}$ Y and Shi L: The requirement of SEPT2 and SEPT7 for migration and invasion in human breast cancer via MEK/ERK activation. Oncotarget 7: 61587-61600, 2016.

8. Zhou J, Lu S, Yang S, Chen H, Shi H, Miao M and Jiao B: MicroRNA-127 post-transcriptionally downregulates Sept7 and suppresses cell growth in hepatocellular carcinoma cells. Cell Physiol Biochem 33: 1537-1546, 2014.

9. Meng X, Zhu Y, Tao L, Zhao S and Qiu S: Periostin has a protective role in melatonin-induced cell apoptosis by inhibiting the eIF2 $\alpha$-ATF4 pathway in human osteoblasts. Int J Mol Med 41: 1003-1012, 2018.

10. Subramaniam M, Jalal SM, Rickard DJ, Harris SA Bolander ME and Spelsberg TC: Further characterization of human fetal osteoblastic hFOB 1.19 and hFOB/ER alpha cells: Bone formation in vivo and karyotype analysis using multicolor fluorescent in situ hybridization. J Cell Biochem 87: 9-15, 2002.

11. Livak KJ and Schmittgen TD: Analysis of relative gene expression data using real-time quantitative P C R and the 2(-Delta Delta C(T)) method. Methods 25: 402-408, 2001.

12. Reiter RJ, Tan DX and Fuentes-Broto L: Melatonin: A multi-tasking molecule. Prog Brain Res 181: 127-151, 2010.

13. Akbarzadeh M, Rahbarghazi R, Nabat E, Movassaghpour AA, Shanehbandi D, Faramarzian Azimi Maragheh B, Matluobi D, Barazvan B, Kazemi M, Samadi N and Nouri M: The impact of different extracellular matrices on melatonin effect in proliferation and stemness properties of ovarian cancer cells. Biomed Pharmacother 87: 288-295, 2017.

14. Bavithra S, Selvakumar K, Sundareswaran L and Arunakaran J: Neuroprotective effect of melatonin against PCBs induced behavioural, molecular and histological changes in cerebral cortex of adult male wistar rats. Neurochem Res 42: 428-438, 2017.

15. Mostowy S and Cossart P: Septins: The forth component of the cytoskeleton. Nat Rev Mol Cell Biol 13: 183-194, 2012.

16. Weirich CS, Erzberger JP and Barral Y: The septin family of GTPases: Architecture and dynamics. Nat Rev Mol Cell Biol 9: 478-489, 2008.

17. Kremer BE, Haystead T and Macara IG: Mammalian septins regulate microtubule stability through interaction with the micro tubule-binding protein MAP4. Mol Biol Cell 16: 4648-4659, 2005.

18. Nagata K and Inagaki M: Cytoskeletal modification of Rho guanine nucleotide exchange factor activity: Identification of a Rho guanine nucleotide exchange factor as a binding partner for Sept9b, a mammalian septin. Oncogene 24: 65-76, 2005.

19. Wloka C, Nishihama R, Onishi M, Oh Y, Hanna J, Pringle JR, Krauss $\mathrm{M}$ and $\mathrm{Bi} \mathrm{E}$ : Evidence that a septin diffusion barrier is dispensable for cytokinesis in budding yeast. Biol Chem 392: 813-829, 2011.

20. Kim MS, Froese CD, Xie H and Trimble WS: Uncovering priciples that control septin-septin interactions. J Biol Chem 287: 30406-30413, 2012.

21. Shehadeh L, Mitsi G, Adi N, Bishopric N and Papapetropoulos S: Expression of lewy body protein septin 4 in postmortem brain of Parkinson's disease and contro subjects. Mov Disord 24: 204-210, 2009.

22. Scott M, Hyland P, McGregor G, Hillan KJ, Russell SE and Hall PA: Multimodality expression profiling shows SEPT9 to be overexpressed in a wide range of human tumours. Oncogene 24: 4688-4700, 2005.

23. Amir S, Wang R, Matzkin H, Simons JW and Mabjeesh NJ: MSF-A interacts with hypoxia-inducible factor-1alpha and augments hypoxia-inducible factor transcriptional activation to affect tumorigenicity and angiogenesis. Cancer Res 66: 856-866, 2006.

24. Horowitz A, Lapointe JF, Eid R, Sheibani S, Gharib N, Jones NK, Vali H, Mandato CA and Greenwood MT: The human septin7 and the yeast CDC10 septin prevent Bax and copper mediated cell death in yeast. Biochim Biophys Acta 1833: 3186-3194, 2013.

25. Jia ZF, Huang Q, Kang CS, Yang WD, Wang GX, Yu SZ, Jiang H and Pu PY: Overexpression of septin 7 suppresses glioma cell growth. J Neurooncol 98: 329-340, 2010.

26. Pizarro JG, Yeste-Velasco M, Esparza JL, Verdaguer E, Pallàs M, Camins A and Folch J: The antiproliferative activity of melatonin in B65 rat dopaminergic neuroblastoma cells is related to the downregulation of cell cycle-related genes. J Pineal Res 45: 8-16, 2008. 